\title{
Comparative Study between Effects of Addition of Fentanyl versus Dexmedetomidine to Local Anesthetic Mixture for Peribulbar Block for Cataract Surgery
}

\author{
Said Mohamed Fayed, Mostafa Mohamed Mahdy, Abdallah Mohamed Ahmed, Amr Mohamed Ahmed \\ Hefny \\ Department of Anaesthesia and ICU, Faculty of Medicine- Al-Azhar University \\ Corresponding author: Amr Mohammed Ahmed Hefny, E-Mail: Amrhefny10@gmail.com, Mobile: 01113385644
}

\begin{abstract}
Background: regional anesthesia is currently more preferable than general anesthesia in ophthalmic surgery and include many types as; peribulbar (extraconal) and retrobulbar (intraconal) anesthesia. Peribulbar block is commonly used because there is reduced risk of globe perforation and optic nerve damage in different to retrobulbar block, but it needs sometimes block supplementations to improve the quality of the block such as clonidine and hyaluronidase. Also, fentanyl and dexmedetomidine was added in this study as an adjuvant to local anesthetics in peribulbar block for cataract surgery.

Objective: the aim of this study was to evaluate and compare the effect of adding fentanyl and dexmedetomidine as adjuvants to local anaesthetic mixture in peribulbar block as regards duration (anesthesia and akinesia), efficacy and pain relief.

Patients and Methods: in this study sixty patients of ASA I-III of both gender, aged 30-60 years arranged into three equal groups (group $\mathrm{S}$, group $\mathrm{F}$ and group $\mathrm{D}$ ) received peribulbar block for phacoemulsification with intraocular lens implantation operation. Group F and group D received fentanyl and dexmedetomidine respectivly with local anaethetics (LA) solution and showed statistically significant difference in onset and duration of globe akinesia respectively compared to group $\mathrm{C}$ which received local anaethetics (LA) solution only.

Results: the onset of globe analgesia was significant in group $\mathrm{F}$ and group D compared to Group S. Postoperative pain was assessed by using a 5-points verbal rating score and recorded data showed significant difference between the three groups with better postoperative pain control in group $\mathrm{F}$ and group $\mathrm{D}$. There was no statistical significant difference between the two groups as regarding the hemodynamics (MBP, HR and $\mathrm{SPO} 2$ ) and the incidence of complications.

Conclusion: the current study concluded that adding fentanyl or Dexmedetomidine to local anaethetics (LA) in peribulbar block significantly reduced the time of onset of globe akinesia and analgesia and reduced the need for second injection. Also, it increased the duration of globe akinesia and analgesia with better postoperative pain control.
\end{abstract}

Keywords: Myocardial infarction, Oxygen saturation - Intravenous

\section{INTRODUCTION}

Regional anesthesia is more preferable than general anesthesia in ophthalmic surgery as most patients are elderly and have chronic diseases as diabetes, hypertention, cardiac and chest problems. Regional anesthesia for eye surgery includes peribulbar and retrobulbar blocks, Peribulbar block is commonly used for ophthalmic surgery in adults ${ }^{(1)}$.

In peribulbar (extraconal) there is reduced risk for globe perforation and optic nerve damage in different to retrobulbar (intraconal) block, However greater volume of anaesthetic solution must be used ${ }^{(2)}$.

Using local anesthetics for peribulbar anesthesia is associated with delayed onset of globe akinesia and corneal anesthesia, short duration of analgesia and sometimes need of block supplementation $^{(3)}$. To decrease the time of onset of action and increase the duration of analgesia, many additives such as clonidine, hyluronidase, sodium bicarbonate and adrenaline, dexmedetomidine were added to local anesthetics ${ }^{(4,5)}$.

Fentanyl (N-phenyl-N-(1-Phenethyl-4piperi-dinyl) propanamide) is an opioid analgesic with potency eighty times that of morphine ${ }^{(4)}$.

Fentanyl is extensively used for anaesthesia and analgesia in the operating room and intensive care unit. It is frequently given intrathecally as a part of spinal anaesthesia or epidurally as a part of epidural anaesthesia and analgesia, it is also used as a sedative ${ }^{(6)}$.Addition of small amount of local anaestheics augments the effect of intrathecal opioids by increasing the duration of the block and speeding the onset of analgesia ${ }^{(7)}$.

Dexmedetomidine was used before in neuroaxial blocks and regional blocks and proved to decrease the onset of anesthesia and akinesia and increase the duration of analgesia and akinesia in all these blocks ${ }^{(8)}$.

This study is designed to examine the effect of adding fentanyl versus dexmedetomidine to local 
anaesthetics in peribulbar block on the onset and duration of lid and globe akinesia and postoperative analgesia comparing them with a control group to detect precisely which one is the best.

\section{AIM OF THE WORK}

The aim of this study is to evaluate and compare the effect of adding fentanyl and dexmedetomidine as adjuvants to local anaesthetic mixture in peribulbar block as regards duration (anesthesia and akinesia), efficacy and pain relief.

\section{PATIENT AND METHODS}

After approval of the Institutional Ethics Committee of Al Azhar University, this prospective, randomized, double-blind control study was conducted in ophthalmology operating theatre, $\mathrm{Al}$ Hussein hospital, Faculty of Medicine, Al Azhar University, in 2018. Sixty patients (aged between 30 and 60 years and ASA I-III) were enrolled in this study and scheduled for elective cataract surgery using peribulbar block, after obtaining written informed consent from each patient.

\section{Eligibility criteria:}

1. Inclusion criteria: Patients aged $30-60$ years old, both sex, ASA physical status I-III, patient with axial globe length below $26 \mathrm{~mm}$.

2. Exclusion criteria: Refusal of the patient to participate in the study, mentally retarded patients and failure of proper communication as in deafness, coagulation abnormalities (INR>1.4), infection at the injection site, preexisting severe cardiovascular or pulmonary disease, renal or hepatic disorder, more than ASA III, high myopia with axial length more than 26 $\mathrm{mm}$, morbidly obese patients (BMI>35), patients with history of hypersensitivity to study drugs.

The enrolled patients were randomized according to computer-generated random number into three equal groups of twenty patients each and concealed using sequentially numbered, sealed opaque envelopes.

The patients will be randomly assigned into 3 equal groups; Group S, Group F and Group D. Control group $\mathrm{S}(\mathrm{n}=20): 5 \mathrm{ml}$ lignocaine $2 \%+3 \mathrm{ml}$ bupivacaine $0.5 \%+1 \mathrm{ml}$ hyaluronidase $(150 \mathrm{IU})+1$ $\mathrm{ml}$ normal saline. Fentanyl group $\mathrm{F}(\mathrm{n}=20): 5 \mathrm{ml}$ lignocaine $2 \%+3 \mathrm{ml}$ bupivacaine $0.5 \%+1$ hyaluronidase $(150 \mathrm{IU})+1 \mathrm{ml}$ fentanyl $(25 \mu \mathrm{g}$ diluted to normal saline $1 \mathrm{ml}$ ) Dexmedetomidine group $\mathrm{D}$ $(\mathrm{n}=20): 5 \mathrm{ml}$ lignocaine $2 \%+3 \mathrm{ml}$ bupivacaine $+1 \mathrm{ml}$ hyaluronidase (150 IU) $+1 \mathrm{ml}$ Dexmedetomidine (25 $\mu \mathrm{g}$ diluted to normal saline $1 \mathrm{ml}$ ).

The study drug solutions were in similar volume of $10 \mathrm{~mL}$, to maintain the blindness of study and were prepared by a resident anesthetist who was not involved for data collection of the patients. The anesthetist performing the block was also blinded to the study groups, and all observations were done by the same investigator.

All patients were admitted before the day of surgery, and fasting of $6 \mathrm{hrs}$ was ensured. On arrival in the preparation room, history was taken from all patients including medical and surgical history, intravenous (IV) cannula was placed and all patients received sedation with midazolam at a fixed dose of $0.02 \mathrm{mg} / \mathrm{kg}$ iv as premedication.

In the operation theater, standard monitoring for noninvasive blood pressure, heart rate, electrocardiogram (ECG), and pulse oximetry $\left(\mathrm{SpO}_{2}\right)$ were commenced to monitor the perioperative vital parameters of patients and nasal $\mathrm{O}_{2}$ cannula was placed.

The patient lied in a supine position and was asked to look directly ahead focusing on a fixed point on the ceiling, so that the eyes were in the neutral position. Peri bulbar block was performed in the specified eye using $10 \mathrm{ml}$ syringe with a $25 \mathrm{G}$ needle for the local anesthetic injection under complete aseptic condition, by inserting the needle at the medial canthus. With sharper bevel facing the globe, the needle was inserted along the medial orbital wall $20 \mathrm{~mm}$ deep, in a perpendicular direction. After negative aspiration, the local anesthetic solution was injected slowly followed by a gentle digital massage to the eyeball to facilitate diffusion of the local anesthetic mixture.

\section{RESULTS}

Table (1): Comparison between groups according to demographic data

\begin{tabular}{|c|c|c|c|c|c|}
\hline Demographic Data & $\begin{array}{c}\begin{array}{c}\text { Group } S \\
(\mathrm{~N}=20)\end{array} \\
\end{array}$ & $\begin{array}{c}\begin{array}{c}\text { Group F } \\
(\mathbf{N}=20)\end{array} \\
\end{array}$ & $\begin{array}{c}\text { Group D } \\
(\mathrm{N}=20)\end{array}$ & $t / x 2$ & p-value \\
\hline $\begin{array}{l}\text { Age (years) } \\
\text { Mean } \pm \text { SD } \\
\text { Range }\end{array}$ & $\begin{array}{c}53.80 \pm 8.68 \\
31-60 \\
\end{array}$ & $\begin{array}{c}51.25 \pm 10.47 \\
32-61\end{array}$ & $\begin{array}{c}52.00 \pm 9.39 \\
30-60\end{array}$ & 0.377 & 0.687 \\
\hline $\begin{array}{l}\text { Sex } \\
\text { Male } \\
\text { Female }\end{array}$ & $\begin{array}{c}5(25.0 \%) \\
15(75.0 \%)\end{array}$ & $\begin{array}{c}9(45.0 \%) \\
11(55.0 \%)\end{array}$ & $\begin{array}{c}8(40.0 \%) \\
12(60.0 \%)\end{array}$ & 1.866 & 0.393 \\
\hline \begin{tabular}{|c|} 
Weight $(\mathbf{k g})$ \\
Mean \pm SD \\
Range \\
\end{tabular} & $\begin{array}{c}87.50 \pm 14.46 \\
60-120 \\
\end{array}$ & \begin{tabular}{|c|}
$79.00 \pm 11.99$ \\
$65-110$ \\
\end{tabular} & $\begin{array}{c}84.00 \pm 11.65 \\
70-110 \\
\end{array}$ & 2.241 & 0.116 \\
\hline $\begin{array}{c}\text { ASA } \\
\text { I } \\
\text { II } \\
\text { III }\end{array}$ & $\begin{array}{c}1(5.0 \%) \\
11(55.0 \%) \\
8(40.0 \%)\end{array}$ & $\begin{array}{c}1(5.0 \%) \\
15(75.0 \%) \\
4(20.0 \%)\end{array}$ & $\begin{array}{c}3(15.0 \%) \\
11(55.0 \%) \\
6(30.0 \%)\end{array}$ & 3.798 & 0.434 \\
\hline
\end{tabular}

t-Independent Sample ANOVA test; p-value >0.05 NS 
Table (2): Comparison between groups according to duration of operation $(\mathrm{min})$

\begin{tabular}{|l|c|c|c|c|c|}
\hline $\begin{array}{c}\text { Duration of } \\
\text { operation }(\mathbf{m i n})\end{array}$ & $\begin{array}{c}\text { Group S } \\
(\mathbf{N}=\mathbf{2 0})\end{array}$ & $\begin{array}{c}\text { Group F } \\
(\mathbf{N}=\mathbf{2 0})\end{array}$ & $\begin{array}{c}\text { Group D } \\
(\mathbf{N}=\mathbf{2 0})\end{array}$ & t-test & p-value \\
\hline Mean \pm SD & $15.00 \pm 2.70$ & $17.15 \pm 2.78$ & $18.75 \pm 2.75$ & 9.528 & $<0.001 * *$ \\
\hline Range & $10-20$ & $15-23$ & $15-25$ & & \\
\hline
\end{tabular}

t-Independent Sample ANOVA test; **p-value $<0.001$ HS

Table (3): Comparison between groups according to heart rate.

\begin{tabular}{|l|c|c|c|c|c|}
\hline Heart rate & $\begin{array}{c}\text { Group S } \\
(\mathbf{N}=\mathbf{2 0})\end{array}$ & $\begin{array}{c}\text { Group F } \\
(\mathbf{N = 2 0})\end{array}$ & $\begin{array}{c}\text { Group D } \\
(\mathbf{N}=\mathbf{2 0})\end{array}$ & t-test & p-value \\
\hline $\begin{array}{l}\text { At 5m } \\
\text { Mean } \pm S D \\
\text { Range }\end{array}$ & $\begin{array}{c}73.10 \pm 9.15 \\
58-90\end{array}$ & $\begin{array}{c}73.10 \pm 9.99 \\
57-94\end{array}$ & $\begin{array}{c}75.85 \pm 14.17 \\
51-100\end{array}$ & 0.394 & 0.676 \\
\hline $\begin{array}{l}\text { At 10m } \\
\text { Mean } \pm S D \\
\text { Range }\end{array}$ & $\begin{array}{c}72.25 \pm 9.28 \\
55-85\end{array}$ & $\begin{array}{c}73.50 \pm 9.30 \\
52-91\end{array}$ & $\begin{array}{c}75.15 \pm 12.93 \\
54-91\end{array}$ & 0.373 & 0.69 \\
\hline $\begin{array}{l}\text { At 15m } \\
\text { Mean } \pm S D\end{array}$ & $\begin{array}{c}74.59 \pm 8.54 \\
\text { Range }\end{array}$ & $\begin{array}{c}74.60 \pm 10.42 \\
59-89\end{array}$ & $\begin{array}{c}75.45 \pm 12.87 \\
52-95\end{array}$ & 0.04 & 0.961 \\
\hline $\begin{array}{l}\text { At 20m } \\
\text { Mean } \pm S D \\
\text { Range }\end{array}$ & $\begin{array}{c}69.50 \pm 0.71 \\
59-78\end{array}$ & $\begin{array}{c}72.11 \pm 12.03 \\
61-88\end{array}$ & $\begin{array}{c}73.00 \pm 11.71 \\
58-93\end{array}$ & 2.093 & 0.147 \\
\hline
\end{tabular}

t-Independent Sample ANOVA test; $p$-value $>0.05$ NS

Table (4): Comparison between groups according to systolic blood pressure ( $\mathrm{mmHg}$ ).

\begin{tabular}{|c|c|c|c|c|c|}
\hline $\begin{array}{c}\text { Systolic blood } \\
\text { pressure (mmHg) }\end{array}$ & $\begin{array}{c}\text { Group S } \\
(\mathrm{N}=\mathbf{2 0})\end{array}$ & $\begin{array}{c}\text { Group F } \\
(\mathrm{N}=\mathbf{2 0})\end{array}$ & $\begin{array}{c}\text { Group D } \\
(\mathrm{N}=\mathbf{2 0})\end{array}$ & t-test & $\begin{array}{c}\text { p- } \\
\text { value }\end{array}$ \\
\hline $\begin{array}{l}\text { At } \mathbf{5 m} \\
\quad \text { Mean } \pm \text { SD } \\
\text { Range }\end{array}$ & $\begin{array}{c}135.80 \pm 10.21 \\
122-160\end{array}$ & $\begin{array}{c}131.65 \pm 9.13 \\
114-145\end{array}$ & $\begin{array}{c}133.55 \pm 8.99 \\
115-144\end{array}$ & 0.965 & 0.387 \\
\hline $\begin{array}{l}\text { At 10m } \\
\text { Mean } \pm \text { SD } \\
\text { Range }\end{array}$ & $\begin{array}{c}135.30 \pm 9.85 \\
118-154\end{array}$ & $\begin{array}{c}131.20 \pm 7.42 \\
117-143 \\
\end{array}$ & $\begin{array}{c}130.60 \pm 12.05 \\
117-173\end{array}$ & 1.322 & 0.275 \\
\hline $\begin{array}{l}\text { At } 15 m \\
\text { Mean } \pm \text { SD } \\
\text { Range } \\
\end{array}$ & \begin{tabular}{|c|}
$130.24 \pm 16.08$ \\
$80-155$
\end{tabular} & $\begin{array}{c}125.60 \pm 5.93 \\
85-151 \\
\end{array}$ & $\begin{array}{c}126.00 \pm 7.39 \\
118-150 \\
\end{array}$ & 1.669 & 0.198 \\
\hline $\begin{array}{l}\text { At } 20 m \\
\text { Mean } \pm \text { SD } \\
\text { Range }\end{array}$ & \begin{tabular}{|c|}
$131.50 \pm 16.26$ \\
$120-143$
\end{tabular} & $\begin{array}{c}133.50 \pm 7.39 \\
124-145\end{array}$ & $\begin{array}{c}124.14 \pm 8.46 \\
111-140\end{array}$ & 3.16 & 0.063 \\
\hline
\end{tabular}

t-Independent Sample ANOVA test; p-value >0.05 NS

Table (5): Comparison between groups according to diastolic blood pressure $(\mathrm{mmHg})$.

\begin{tabular}{|c|c|c|c|c|c|}
\hline $\begin{array}{c}\text { Diastolic blood } \\
\text { pressure (mmHg) }\end{array}$ & $\begin{array}{c}\text { Group S } \\
(\mathrm{N}=\mathbf{2 0})\end{array}$ & $\begin{array}{c}\text { Group F } \\
(\mathrm{N}=20)\end{array}$ & $\begin{array}{c}\text { Group D } \\
(\mathbf{N}=20)\end{array}$ & t-test & $\begin{array}{c}\text { p- } \\
\text { value }\end{array}$ \\
\hline \begin{tabular}{|l|} 
At 5m \\
Mean \pm SD \\
Range \\
\end{tabular} & $\begin{array}{c}74.70 \pm 9.25 \\
62-100 \\
\end{array}$ & $\begin{array}{c}69.05 \pm 4.56 \\
59-75 \\
\end{array}$ & $\begin{array}{c}70.45 \pm 5.04 \\
64-81 \\
\end{array}$ & 1.693 & 0.212 \\
\hline $\begin{array}{l}\text { At 10m } \\
\text { Mean } \pm \text { SD } \\
\text { Range }\end{array}$ & $\begin{array}{c}72.75 \pm 9.96 \\
58-95\end{array}$ & $\begin{array}{c}68.00 \pm 4.50 \\
55-73\end{array}$ & $\begin{array}{c}70.60 \pm 7.11 \\
63-90\end{array}$ & 1.266 & 0.091 \\
\hline $\begin{array}{l}\text { At 15m } \\
\text { Mean } \pm \text { SD } \\
\text { Range } \\
\end{array}$ & $\begin{array}{c}70.47 \pm 6.43 \\
60-80 \\
\end{array}$ & $\begin{array}{c}67.65 \pm 4.07 \\
55-71 \\
\end{array}$ & $\begin{array}{c}68.10 \pm 7.29 \\
56-87 \\
\end{array}$ & 2.128 & 0.085 \\
\hline $\begin{array}{l}\text { At 20m } \\
\text { Mean } \pm \text { SD } \\
\text { Range }\end{array}$ & $\begin{array}{c}67.00 \pm 5.66 \\
63-71\end{array}$ & $\begin{array}{c}66.50 \pm 3.82 \\
60-71\end{array}$ & $\begin{array}{c}64.64 \pm 4.57 \\
53-73\end{array}$ & 0.589 & 0.564 \\
\hline
\end{tabular}

t-Independent Sample ANOVA test; p-value >0.05 NS

Table (6): Comparison between groups according to $\mathrm{SpO}_{2}$.

\begin{tabular}{|l|c|c|c|c|c|}
\hline $\mathbf{S p O}_{2}$ & $\begin{array}{c}\text { Group S } \\
(\mathbf{N}=\mathbf{2 0})\end{array}$ & $\begin{array}{c}\text { Group F } \\
(\mathbf{N}=\mathbf{2 0})\end{array}$ & $\begin{array}{c}\text { Group D } \\
(\mathbf{N}=\mathbf{2 0})\end{array}$ & ANOVA & $\begin{array}{c}\text { p- } \\
\text { value }\end{array}$ \\
\hline At 5m & $98.84 \pm 0.96$ & $98.84 \pm 0.75$ & $98.49 \pm 0.91$ & 1.479 & 0.157 \\
\hline At 10m & $98.59 \pm 1.02$ & $98.79 \pm 0.95$ & $98.89 \pm 0.81$ & 1.193 & 0.083 \\
\hline At 15m & $98.84 \pm 0.75$ & $98.59 \pm 1.02$ & $98.89 \pm 1.02$ & 0.818 & 0.389 \\
\hline At 20m & $98.59 \pm 1.02$ & $98.84 \pm 0.75$ & $98.54 \pm 0.95$ & 0.102 & 0.676 \\
\hline
\end{tabular}

Table (6): Comparison between groups according to onset (min).

\begin{tabular}{|c|c|c|c|c|c|}
\hline Onset (Min) & $\begin{array}{c}\text { Group S } \\
(\mathbf{N}=20)\end{array}$ & $\begin{array}{c}\text { Group F } \\
(\mathbf{N}=\mathbf{2 0})\end{array}$ & $\begin{array}{c}\text { Group D } \\
(\mathbf{N}=20)\end{array}$ & t-test & p-value \\
\hline $\begin{array}{c}\text { Sensory block } \\
\text { Mean } \pm \text { SD } \\
\text { Range }\end{array}$ & $\begin{array}{c}2.35 \pm 0.83 \\
1-4\end{array}$ & $\begin{array}{c}1.73 \pm 0.57 \\
1-3\end{array}$ & $\begin{array}{c}0.83 \pm 0.34 \\
0.5-1.5\end{array}$ & 31.271 & $<0.001^{* *}$ \\
\hline $\begin{array}{c}\text { Globe akinsia } \\
\text { Mean } \pm \text { SD } \\
\text { Range }\end{array}$ & $\begin{array}{c}4.70 \pm 1.98 \\
2-8\end{array}$ & $\begin{array}{c}4.20 \pm 1.28 \\
2-6\end{array}$ & $\begin{array}{c}7.10 \pm 1.89 \\
4-10\end{array}$ & 15.819 & $<0.001^{* *}$ \\
\hline
\end{tabular}

t-Independent Sample ANOVA test; **p-value $<0.001$ HS

Table (7): Comparison between groups according to duration ( $\mathrm{min})$.

\begin{tabular}{|l|c|c|c|c|c|}
\hline Duration $(\mathbf{M i n})$ & $\begin{array}{c}\text { Group S } \\
(\mathbf{N}=20)\end{array}$ & $\begin{array}{c}\text { Group F } \\
(\mathbf{N}=20)\end{array}$ & $\begin{array}{c}\text { Group D } \\
(\mathbf{N}=20)\end{array}$ & t-test & p-value \\
\hline $\begin{array}{l}\text { Sensory block } \\
\text { Mean } \pm \text { SD }\end{array}$ & $\begin{array}{c}147.50 \pm 18.03 \\
\text { Range }\end{array}$ & $\begin{array}{c}197.90 \pm 20.30 \\
166-244\end{array}$ & $\begin{array}{c}246.95 \pm 33.51 \\
200-310\end{array}$ & 79.77 & $<0.001^{* * *}$ \\
\hline $\begin{array}{l}\text { Globe akinsia } \\
\text { Mean } \pm \text { SD } \\
\text { Range }\end{array}$ & $\begin{array}{c}144.00 \pm 23.26 \\
100-180\end{array}$ & $\begin{array}{c}295.65 \pm 32.50 \\
212-356\end{array}$ & $\begin{array}{c}168.50 \pm 14.88 \\
150-190\end{array}$ & 218.669 & $<0.001^{* *}$ \\
\hline
\end{tabular}

$\mathrm{t}$-Independent Sample ANOVA test; **p-value $<0.001 \mathrm{HS}$

Table (8): Comparison between groups according to postoperative analgesia.

\begin{tabular}{|c|c|c|c|c|c|}
\hline Postoperative analgesia & $\begin{array}{c}\text { Group S } \\
(\mathrm{N}=20)\end{array}$ & $\begin{array}{c}\text { Group F } \\
(\mathrm{N}=20)\end{array}$ & $\begin{array}{c}\text { Group D } \\
(\mathrm{N}=20)\end{array}$ & $x^{2}$ & p-value \\
\hline $\begin{array}{l}\text { Immediately after surgery } \\
\text { No pain } \\
\text { Mild pain } \\
\text { Moderate } \\
\text { Severe } \\
\text { Unbearable pain }\end{array}$ & \begin{tabular}{|c|}
$20(100.0 \%)$ \\
$0(0.0 \%)$ \\
$0(0.0 \%)$ \\
$0(0.0 \%)$ \\
$0(0.0 \%)$
\end{tabular} & $\begin{array}{c}20(100.0 \%) \\
0(0.0 \%) \\
0(0.0 \%) \\
0(0.0 \%) \\
0(0.0 \%)\end{array}$ & $\begin{array}{c}20100.0 \%) \\
0(0.0 \%) \\
0(0.0 \%) \\
0(0.0 \%) \\
0(0.0 \%)\end{array}$ & 0.000 & 1.000 \\
\hline \begin{tabular}{|l|} 
Pain score After $1 \mathbf{h r}$ \\
No pain \\
Mild pain \\
Moderate \\
Severe \\
Unbearable pain \\
\end{tabular} & $\begin{array}{c}18(90.0 \%) \\
2(10.0 \%) \\
0(0.0 \%) \\
0(0.0 \%) \\
0(0.0 \%) \\
\end{array}$ & \begin{tabular}{|c|}
$20(100.0 \%)$ \\
$0(0.0 \%)$ \\
$0(0.0 \%)$ \\
$0(0.0 \%)$ \\
$0(0.0 \%)$ \\
\end{tabular} & $\begin{array}{c}20(100.0 \%) \\
0(0.0 \%) \\
0(0.0 \%) \\
0(0.0 \%) \\
0(0.0 \%) \\
\end{array}$ & 4.138 & 0.126 \\
\hline \begin{tabular}{|l|} 
Pain score After $2 \mathrm{hrs}$ \\
No pain \\
Mild pain \\
Moderate \\
Severe \\
Unbearable pain \\
\end{tabular} & $\begin{array}{c}14(70.0 \%) \\
4(20.0 \%) \\
2(10.0 \%) \\
0(0.0 \%) \\
0(0.0 \%) \\
\end{array}$ & $\begin{array}{c}18(90.0 \%) \\
2(10.0 \%) \\
0(0.0 \%) \\
0(0.0 \%) \\
0(0.0 \%) \\
\end{array}$ & $\begin{array}{c}19(95.0 \%) \\
1(5.0 \%) \\
0(0.0 \%) \\
0(0.0 \%) \\
0(0.0 \%) \\
\end{array}$ & 6.824 & 0.146 \\
\hline \begin{tabular}{|l|} 
Pain score After $\mathbf{6 h r s}$ \\
No pain \\
Mild pain \\
Moderate \\
Severe \\
Unbearable pain \\
\end{tabular} & $\begin{array}{c}13(65.0 \%) \\
4(20.0 \%) \\
2(10.0 \%) \\
1(5.0 \%) \\
0(0.0 \%) \\
\end{array}$ & $\begin{array}{c}17(85.0 \%) \\
2(10.0 \%) \\
1(5.0 \%) \\
0(0.0 \%) \\
0(0.0 \%) \\
\end{array}$ & $\begin{array}{c}14(70.0 \%) \\
3(15.0 \%) \\
3(15.0 \%) \\
0(0.0 \%) \\
0(0.0 \%)\end{array}$ & 4.258 & 0.642 \\
\hline $\begin{array}{l}\text { Pain score After 12hrs } \\
\text { No pain } \\
\text { Mild pain } \\
\text { Moderate } \\
\text { Severe } \\
\text { Unbearable pain }\end{array}$ & $\begin{array}{c}3(15.0 \%) \\
10(50.0 \%) \\
5(25.0 \%) \\
210.0 \%) \\
0(0.0 \%)\end{array}$ & $\begin{array}{c}0(0.0 \%) \\
12(60.0 \%) \\
7(35.0 \%) \\
1(5.0 \%) \\
0(0.0 \%)\end{array}$ & $\begin{array}{c}0(0.0 \%) \\
10(50.0 \%) \\
10(50.0 \%) \\
0(0.0 \%) \\
0(0.0 \%)\end{array}$ & 9.977 & 0.126 \\
\hline
\end{tabular}

$\mathrm{x} 2$ : Chi-square test; $\mathrm{p}$-value $>0.05 \mathrm{NS}$

Table (9): Comparison between groups according to patient satisfaction.

\begin{tabular}{|l|c|c|c|c|c|}
\hline $\begin{array}{l}\text { Patient } \\
\text { satisfaction }\end{array}$ & $\begin{array}{c}\text { Group S } \\
(\mathbf{N}=\mathbf{2 0})\end{array}$ & $\begin{array}{c}\text { Group F } \\
(\mathbf{N}=\mathbf{2 0})\end{array}$ & $\begin{array}{c}\text { Group D } \\
(\mathbf{N}=\mathbf{2 0})\end{array}$ & t-test & $\begin{array}{c}\text { p- } \\
\text { value }\end{array}$ \\
\cline { 1 - 4 } Mean \pm SD & $\begin{array}{c}3.60 \pm 0.5 \\
0\end{array}$ & $\begin{array}{c}3.95 \pm 0.2 \\
2\end{array}$ & $\begin{array}{c}3.95 \pm 0.2 \\
2\end{array}$ & $\begin{array}{c}6.94 \\
8\end{array}$ & \multirow{2}{*}{$0.002^{*}$} \\
\cline { 1 - 3 } Range & $3-4$ & $3-4$ & $3-4$ & & \\
\hline
\end{tabular}

t-Independent Sample ANOVA test; * ${ }^{p}$-value $<0.05 \mathrm{~S}$ 
Table (10): Comparison between groups according to surgeon satisfaction.

\begin{tabular}{|l|c|c|c|c|c|}
\hline Surgeon satisfaction & $\begin{array}{c}\text { Group S } \\
(\mathbf{N}=\mathbf{2 0})\end{array}$ & $\begin{array}{c}\text { Group F } \\
(\mathbf{N}=\mathbf{2 0})\end{array}$ & $\begin{array}{c}\text { Group D } \\
(\mathbf{N}=\mathbf{2 0})\end{array}$ & t-test & p-value \\
\hline Mean \pm SD & $3.70 \pm 0.47$ & $3.80 \pm 0.52$ & $3.85 \pm 0.37$ & & \multirow{2}{*}{0.556} \\
\hline Range & $3-4$ & $2-4$ & $3-4$ & 0.576 \\
\hline
\end{tabular}

t-Independent Sample ANOVA test; p-value >0.05 NS

Table (11): Comparison between groups according to complications of the used drugs.

\begin{tabular}{|l|c|c|c|c|c|}
\hline $\begin{array}{c}\text { Complications of } \\
\text { the used drugs }\end{array}$ & $\begin{array}{c}\text { Group S } \\
(\mathbf{N}=\mathbf{2 0})\end{array}$ & $\begin{array}{c}\text { Group F } \\
(\mathbf{N}=\mathbf{2 0})\end{array}$ & $\begin{array}{c}\text { Group D } \\
(\mathbf{N}=\mathbf{2 0})\end{array}$ & $\mathbf{x}^{\mathbf{2}}$ & p-value \\
\hline No & $17(85.0 \%)$ & $18(90.0 \%)$ & $19(95.0 \%)$ & \multirow{2}{*}{1.111} & \multirow{2}{*}{0.574} \\
\hline Yes & $3(15.0 \%)$ & $2(10.0 \%)$ & $1(5.0 \%)$ & \\
\hline
\end{tabular}

$\mathrm{x}^{2}$ : Chi-square test; $\mathrm{p}$-value $>0.05 \mathrm{NS}$

\section{DISCUSSION}

In the current study, we demonstrated the effect of adding fentanyl vs dexmedetomidine to LA mixture for patients undergoing peribulbar anesthesia for phacoemulsification with intraocular lens implantation surgery, the study was held in the ophthalmic operating theatre at $\mathrm{Al}$ Hussein Hospital, Al Azhar University. Sixty patients, their age ranging from 30 to 60 years, ASA I-III, were enrolled in the form of three groups, Group $S$ (n $=20$ ) the peribulbar block was performed using a local anesthetic mixture alone, Group F $(n=20)$ the peribulbar block was performed using a local anesthetics mixture plus fentanyl and Group D (n $=20$ ) the peribulbar block was performed using a local anesthetic mixture plus dexmedetomidine.

There was no significant difference between the three groups (group $\mathrm{S}$, group $\mathrm{F} \&$ group $\mathrm{D}$ ) regarding the demographic characteristics, hemodynamic changes (MBP \& $\mathrm{HR} \& \mathrm{SPO}_{2}$ ) and incidence of complications.

However, Adding opioid analgesic (fentanyl) or Alfa 2 Agonist (dexmedetomidine)to local anesthetic mixture was effective on improving the quality of the block as evidenced by accelerating the onset and increasing the duration of globe akinesia and analgesia and reducing the postoperative pain with low pain scores with different degree.

\section{Regarding Fentanyl:}

Study has demonstrated the presence of peripheral opioid receptors that mediate analgesia by endogenous as well as exogenous opioid agonists ${ }^{(9)}$. It is speculated that the peripheral administration of opioids provides stronger and longer lasting analgesia with a lower dose of opioid without central side effects such as respiratory depression, nausea, vomiting and pruritus. A number of trials have examined the peripheral analgesic effect of opioids in a large variety of surgical settings particularly arthroscopy and conduction nerve blocks ${ }^{(10)}$.

Fentanyl is reported to have a local anaesthetic action $^{(11)}$.

Gormley et al. ${ }^{(12)}$ suggested that alfentanil also prolonged postoperative analgesia by local anaesthetic action.

Also, fentanyl may potentiate local anaesthetic action via central opioid receptormediated analgesia by peripheral uptake of fentanyl to systemic circulation ${ }^{(13)}$.

A synergistic interaction between local anaesthetics and opioids with epidural administration has been reported $^{(14)}$. It appears that local anaesthetics and opioids exert their action independently via different mechanisms. Local anaesthetics block propagation and generation of neural action potentials by a selective effect on sodium channels, whereas opioids act on the opioid receptors creating an increase in a potassium conductance. This action results in hyperpolarization of then nerve cell membrane and a decrease in excitability. Although sodium channel block is proposed to be the primary mode of action, local anaesthetics also have an effect on synaptic transmission ${ }^{(15)}$.

Li et al. ${ }^{(15)}$ showed that lidocaine inhibited both substances $\mathrm{P}$ binding and substance $\mathrm{P}$-evoked increase in intracellular calcium. In contrast, in addition to the considered primary mode of action, opioids were found to directly suppress the action potential in nerve fibers.

Frazier et al $^{(16)}$ showed that morphine depressed both sodium and potassium currents associated with the action potential in squid giant axons. Therefore, the combination of local anaesthetics and opioids may effectively inhibit multiple areas of neuronal excitability.

Kumar et $\boldsymbol{a l} .{ }^{(17)}$ found that wound infiltration with fentanyl has lower visual analogue pain score (VAS) postoperatively and combination of lidocaine with fentanyl for wound infiltration in cholecystectomy patients was associated with better postoperative analgesia, reduced analgesic consumption and better lung function.

Saryazdi et al. ${ }^{(18)}$ found that injection of fentanyl intraarticularly has better postoperative pain less pain score and short time to walk were achieved by fentanyl orpethedine in comparison with dexamethasone when injected intraarticular. 


\section{Regarding Dexmedetomidine:}

The results of this study showed that addition of 25 micg dexmedetomidine to a mixture of lidocaine $2 \%$ and bupivacaine $0.5 \%$ to peribulbar block prolonged the duration of sensory and motor akinesia in a statistically significant way. Peribulbar block is commonly used for phacoemulsification with intraocular lens implantation surgery, but using only local anesthetics for peribulbar anesthesia is usually associated with delayed onset of globe akinesia and corneal anesthesia, short duration of analgesia and frequent need of block supplementation of analgesia, many additives such as adrenaline, sodium bicarbonate and clonidine were added to local anesthetics. Dexmedetomidine is centrally acting, highly specific $\alpha 2$ agonist, the exact mechanism by which $\alpha 2$ adrenergic receptor agonists produce analgesia and sedation is not fully understood but it is likely to be multifactorial ${ }^{(19)}$. Peripherally, $\alpha 2$-agonists reduce the release of norepinephrine and cause $\alpha 2$ receptor independent inhibitory effects on nerve fiber action potentials. Centrally, $\alpha 2$ agonists inhibit substance $\mathrm{P}$ in the nociceptive pathway at the level of the dorsal root neuron and activate $\alpha 2$ adrenoceptors in the locus coeruleus ${ }^{(19)}$. So, activation of the $\alpha 2$ receptors will inhibit the release of norepinephrine, terminating the propagation of pain signals. Postsynaptic activation of $\alpha 2$ adrenoceptors in the central nervous system inhibits the sympathetic activity and thus can decrease the blood pressure and HR.

Channabasappa et al. ${ }^{(20)}$ evaluated the effect of adding two different doses $(25 \mathrm{micg}$ and $50 \mathrm{micg}$ ) of dexmedetomidine to the lidocaine/bupivacaine mixture for peribulbar block in cataract surgeries. They reported that the onset of corneal anesthesia and globe akinesia was significantly shorter in group D50 than in the control group. In group D25, the onset of corneal anesthesia was significantly shorter than the control group but not the globe akinesia. These findings were coinciding with the results of this study regarding the onset of corneal anesthesia and globe akinesia in group D25.

Whereas in another study by Hala et al. ${ }^{(21)}$, who studied the effect of adding two different doses of dexmedetomidine (25 micg and $50 \mathrm{micg}$ ) to levobupivacaine/hyaluronidase mixture and assessed their effects on the onset and duration of globe anesthesia and akinesia, they found out that the onset of comeal anesthesia and globe akinesia in group D25 was shorter than in the control group but the difference was not statistically significant.

Madan et al. $^{(22)}$, compared the effect of clonidine added to lidocaine/hyaluronidase mixture in peribulbar block in three different doses $(0.5 \mathrm{micg} / \mathrm{kg}, 1$ micg/ $/ \mathrm{kg}$ and $1.5 \mathrm{micg} / \mathrm{kg}$ ) at the onset and duration of globe anesthesia and akinesia. They found that there was no statistically significant difference in between the three groups or when comparing each group to the control group regarding the onset of globe anesthesia or akinesia.

Reem et al. ${ }^{(23)}$ compared the two $\alpha 2$ agonists (clonidine versus dexmedetomidine) in peribulbar block. They studied two groups of patients: one group received a mixture of lidocaine/bupivacaine $1 \mathrm{micg} / \mathrm{kg}$ clonidine and the other received lidocaine/bupivacaine $1 \mathrm{micg} / \mathrm{kg}$ dexmedetomidine. There was no statistical significance between the two groups regarding the onset of globe anesthesia and akinesia.

Also, Madan et al's ${ }^{(22)}$ study found that the duration of globe analgesia and akinesia was significantly prolonged in patients receiving 1.0 and $1.5 \mathrm{micg} / \mathrm{kg}$ clonidine with lidocaine/ hyaluronidase mixture when compared to the patients who received the lidocaine/hyaluronidase mixture alone. In Reem et al's ${ }^{(23)}$ study, the duration of globe analgesia and globe akinesia showed a statistically significant increase in both groups of study (the clonidine group and dexmedetomidine group) in comparison with the control group.

\section{CONCLUSION}

The current study concluded that adding Fentanyl or Dexmedetomidine to local anaethetics (LA) in peribulbar block significantly reduced the time of onset of globe akinesia and analgesia and reduced the need for second injection. Also, it increased the duration of globe akinesia and analgesia with better postoperative pain control. Also, it was noticed that adding fentanyl or Dexmedetomidine to local anesthetic solution did not significantly affect the Patients hemodynamics, with no block related complications, so fentanyl or Dexmedetomidine was considered safe and effective adjuvant to local anaethetics (LA).

\section{REFERENCES}

1. Stead SW, Beatie DB, Keyes MA (1998): Anesthesia for ophthalmic surgery. Principles and practice of anesthesiology, 5:2181-99.

2. Sanford DK, Minoso y de Cal OE, Belyea DA (1998): Response of intraocular pressure to Retrobulbar and peribulbar anesthesia. Ophthalmic Surg Lasers, 29:815-817.

3. Rubin AP (1995): Complications of local anaesthesia for ophthalmic surgery. $\mathrm{Br} \mathrm{J}$ Anaesth., 75:93-6. 
4. Zahl K, Jordan A, McGroarty J et al. (1990): $\mathrm{pH}$-adjusted bupivacaine and hyaluronidase for peribulbar block. Anesthesiology, 72:230-2.

5. Reah G, Bodenham AR, Braithwaite P, Esmond J, Menage MJ (1998): Peribulbar anesthesia using a mixture of local anaesthetic and vecuronium. Anaesthesia, 53:551-

6. Barr pharmaceuticals (2006): Barr Launches Generic ACTIQR Cancer pain management product. https:// www. biospace. com/.../ releases/barr-pharmaceuticals-inc-launchesgeneric-actiq.

7. Palmer CM, Van Maren G, Nogami WM, Alves D (1999): Bupivacine augments intrathecal fentanyl for labour analgesia. Anesthesiology, 91:84-9.

8. Channabasappa SM, Shetty VR, Dharamppa SK, Sarma J (2013): Efficacy and safety of dexmedetomidine as an additive to local anesthetics in peribulbar block for cataract surgery. Anesth Essays Res., 7:39-43.

9. Stein C, Millan MJ, Shippenberg TS, Peter K, Herz A (1989): Peripheral opioid receptors mediating antinociception in inflammation. Evidence for involvement of mu, delta and kappa receptors. Journal of Pharmacology and Experimental Therapeutics, 248:1269-1275.

10. Tverskoy M, Braslavsky A, Mazor A, Ferman R, Kissin I (1998): The peripheral effect of fentanyl on postoperative pain. Anesth Analg., 87:1121-4.

11. Gissen AJ, Gugino LD, Datta S et al. (1987): Effects of fentanyl and sufentanil on peripheral mammalian nerves. Anesth Analg., 66:1272-6.

12. Gormley WP, Murray JM, Fee JPH, Bower $S$ (1996): Effect of the addition of alfentanil to lignocaine during axillary brachial plexus anaesthesia. Br J Anaesth., 76:802-5.

13. Nishikawa K, Kanaya $N$, Nakayama $M$ et al. (2000): Fentanyl improves analgesia but prolongs the onset of axillary brachial plexus block by peripheral mechanism. Anesth Analg., 91:384-387.

14. Vercauteren $M$ and Meert TF (1997): Isobolographic analysis of the interaction between epidural sufentanil and bupivacaine in rats. Pharmacol Biochem Behav., 58:237-42.

15. Li YM, Wingrove DE, Too P et al. (1995): Local anaesthetics inhibit substance $P$ binding and evoked increases in intracellular $\mathrm{Ca} 2+$. Anesthesiology, 82:166-73.
16. Frazier DT, Murayama K, Abbott NJ et al. (1972): Effects of morphine on internally perfused squid axons. Proc Soc Exp Biol Med., 139:434-8.

17. Kumar VP, Bhardwaj N, Sharma K, Batra YK (2006): Peripheral analgesic effect of wound infiltration with lignocaine, fentanyl and combination of lignocaine-fentanyl on post operative pain. Journal of Anaesthesiology Clinical Pharmacology, 22(2): 161-165.

18. Saryazdi H, Kashefi P, Heydari M, Kiani A (2006): Analgesic effect of intraarticular fentanyl, pethedine and dexamethasone after knee arthroscopic surgery. Journal of Research in Medical Science, 11:156-159.

19. Eisenach JC, De Kock M, Klimscha W (1996): Alpha (2)-adrenergic agonists for regional anesthesia. A clinical review of clonidine (1984-1995). Anesthesiology, 85: 655-74.

20. Channabasappa SM, Shetty VR, Dharamppa SK, Sarma J (2013): Efficacy and safety of dexmedetomidine as an additive to local anesthetics in peribulbar block for cataract surgery. Anesth Essays Res., 7:39-43.

21. El-Ozairy Hala S, Tharwat Ayman I (2014): Comparative study of the effect of adding two different doses of dexmedetomidine to levobupivacaine/ hyaluronidase mixture on the peribulbar block in vitreoretinal surgery. AinShams J Anesthesiol., 7: 393-9.

22. Madan R, Bharti N, Shende D et al. (2001): A dose response study of clonidine with local anaesthetic mixture for peribulbar block: a comparison of three doses. Anesth Analg., 93:1593.

23. El Kabarity Reem H, Khashaba Mohamed Y (2012): Dexmedetomidine versus clonidine as an adjuvant to peribulbar block in vitreoretinal surgery. Ain-Shams J Anesthesiol., 5:288-94. 